\title{
Low Field Strength Magnetic Resonance Imaging
}

National Cancer Institute

\section{Source}

National Cancer Institute. Low Field Strength Magnetic Resonance Imaging. NCI

Thesaurus. Code C116456.

A type of magnetic resonance imaging that uses a weaker magnet (less than or equal to 0.3 Tesla) than is used in conventional magnetic resonance imaging. This is typical of open MRI machines and office-based MRI units. 